\title{
An Intercell Interference Coordination Scheme in LTE Downlink Networks based on User Priority and Fuzzy Logic System
}

\author{
A. Daeinabi ${ }^{1}$, K. Sandrasegaran ${ }^{1}$, and X.Zhu ${ }^{2}$ \\ Centre for Real-time Information Networks, \\ School of Computing and Communications, Faculty of Engineering and Information \\ Technology, University of Technology Sydney, Sydney, Australia \\ Ameneh. Daeinabi@student.uts.edu.au, \\ Kumbesan. Sandrasegaran@uts. edu . au \\ School of Information and Communications, Beijing University of Posts and \\ Telecommunications Beijing, China \\ zhuxn@bupt . edu. cn
}

\begin{abstract}
The Intercell Interference (ICI) problem is one of the main challenges in Long Term Evolution (LTE) downlink system. In order to deal with the ICI problem, this paper proposes a joint resource block and transmit power allocation scheme in LTE downlink networks. The proposed scheme is implemented in three phases: (1) the priority of users is calculated based on interference level, Quality of Service (QoS) and Head of Line (HoL) delay;(2) users in each cell are scheduled on the specified subbands based on their priority; and (3) eNodeBs dynamically control the transmit power using a fuzzy logic system and exchanging messages to each other. Simulation results demonstrate that the proposed priority scheme outperforms the existing Reuse Factor one (RF1) and Soft Frequency Reuse (SFR) schemes in terms of cell throughput, cell edge user throughput, delay and interference level.
\end{abstract}

\section{KEYWORDS}

LTE, intercell interference coordination, resource block allocation, transmit power allocation, fuzzy logic.

\section{INTRODUCTION}

In recent years, the demand for mobile broadband services with higher data rates and better Quality of Service (QoS) is growing rapidly and this demand has motivated 3GPP to work on Long Term Evolution (LTE). One of their main goals was to define a simple protocol which involves both the radio access network (RAN) and the network core [1]. Moreover, it can obtain the peak rates of $100 \mathrm{Mb} / \mathrm{s}$ and a radio network delay of less than $5 \mathrm{~ms}$, improve the spectrum efficiency and support the flexible bandwidth. In addition, the new flat network architecture can reduce the latency rather than $3 \mathrm{G}$ [2]. The multiple access technologies on the air interface are different in downlink and uplink of LTE systems; Orthogonal Frequency Division Multiple Access (OFDMA) is the downlink multiple access technology while for uplink, Single Carrier Frequency Division Multiple Access (SC-FDMA) is deployed [3]. Moreover, LTE supports frequency division duplex (FDD), time division duplex (TDD) as well as the wide range of system bandwidths which enables the system to work in a great number of different spectrum allocations [4].

DOI : 10.5121/ijwmn.2013.5404 
Since the radio bandwidth is one of the scarce resources in wireless networks, new resource allocation algorithms need to be introduced to overcome radio resource limitation particularly when applications with high data rate are deployed. For this purpose, frequency reuse one has been used in cellular networks. However, the system performance is severely degraded due to increase of interference caused by neighbouring cells. There are two major categories of interference for cellular mobile communication system: intracell interference and intercell interference. Since the LTE downlink systems use OFDMA [5], the orthogonality among subcarriers is designed to mitigate the intracell interference. However, the intercell interference (ICI) caused by using the same frequency in neighbouring cells, can restrict the LTE performance in terms of throughput and spectral efficiency, particularly for cell edge users (CEU). Note that CEUs are user equipments (UEs) in a cell which are far from the serving eNodeB (eNB) while cell centre users (CCUs) are close to the serving eNB. Therefore, the ICI mitigation is a critical point to improve the performance of the system.

In this paper, we consider a dynamic priority based scheme in which the resource allocation for each subband is performed based on user priority. In addition, the transmit power of each Resource Block (RB) is dynamically determined through a fuzzy logic system. Note that a RB is defined as a smallest radio resource which is allocated to a UE. In addition, the system traffic loads as well as system changes are taken into account when the RBs and transmit powers are allocated to different UEs. The proposed scheme could jointly optimize RB and power allocation for each cell, while in the traditional methods, the spectrum allocation or the power allocation is fixed in each cell. In addition, the proposed scheme is a decentralized scheme in which each eNB specifies its own RB allocation and transmit power by exchanging messages with neighbouring eNBs over X2 interface. Consequently, it could improve the system performance in terms of cell throughput and cell edge throughput.

The rest of the paper is organized as follow. The ICI formulation is provided in Section 2. In Section 3, the related work is reviewed. In Section 4, the proposed ICIC scheme is described. Simulation results are presented in Section 5. The conclusion is given in the final Section.

\section{INTERCELL INTERFERENCE FORMULATION}

When a UE moves away from the serving eNB and becomes closer to the neighbouring eNB, the strength of desired received signal decreases and the ICI increases. The impact of ICI in LTE downlink can be analysed using the received Signal to Interference and Noise Ratio (SINR) of $\mathrm{UE} m$ on $\mathrm{RB} n$ as given in (1).

$$
\operatorname{SIN}_{m, n}=\frac{p_{n}^{l} H_{m, n}^{l}}{\sum_{k \neq l} p_{n}^{k} H_{m, n}^{k} \delta_{n}^{k}+P_{N}}
$$

where $P_{n}^{l}$ and $P_{n}^{k}$ are the transmit power from the serving cell $l$ and neighbouring cell $k$ on $\mathrm{RB} n$, respectively. Moreover, $H_{m, n}^{l}$ and $H_{m, n}^{k}$ denote the channel gain from the cell $l$ and cell $k$ to UEm on $\mathrm{RB} n$, and $P_{N}$ is the noise power. $\delta_{n}^{k}$ is set to 1 or 0 to indicate whether the neighbouring cell $k$ allocates $\mathrm{RB} n$ to its UEs or not. It can be summarised that three important factors have a significant influence on the SINR of each UE namely the channel gain from eNB to UE, transmit power on each RB and RB allocation scheme.

From (1), it can be observed that the SINR of UEs closer to their serving eNB is higher compared to the UEs further away from their serving eNB. Furthermore, UEs at the cell boundary would experience higher interference while the desired signal is relatively low. This situation greatly affects the SINR value and the supportable services to users at the cell edge area. As a result, in many ICI mitigation schemes, UEs are classified into CEU and CCU. For different UE 


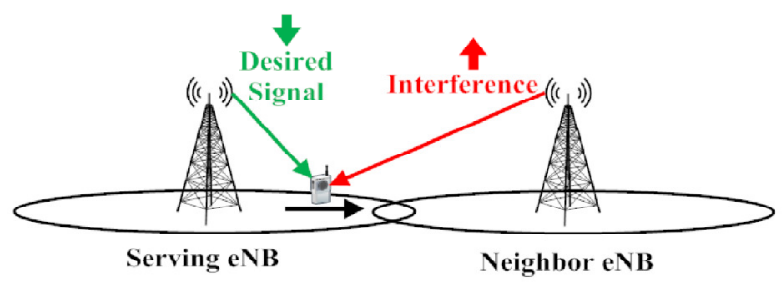

Figure 1. Illustration of UE moving away from its serving eNB

classifications (i.e. either CEU or CCU), alternative power and frequency allocation schemes can be adopted. This allows each UE, particularly CEU, increasing the transmit power which leads to a conflict over the system performance. More specifically, transmitting at higher power for CEU may marginally improve the SINR but results in a significant increase of overall interference in the system. Consequently, the higher transmit power impacts the actual overall system performance of all UEs. Therefore, in order to improve the system performance, a number of power allocation schemes are proposed to achieve an optimal compromise between the achieved SINR and the resulting interference. The third factor turns the ICI mitigation scheme into a variety of frequency or RB allocation algorithms in which the objective of optimization is to reduce the ICI and achieve higher spectral efficiency simultaneously, especially for the CEUs. From (1), it can be seen that the interference can significantly decrease when the serving cell transmits data on $\mathrm{RB} n$ if the neighbouring cells do not allocate the $\mathrm{RB} n$ to their UEs, (i.e.,

). The granularity of the allocation can be a RB or a portion of the available bandwidth. The concept to reserve parts of the bandwidth to avoid interference is classified as a frequency reuse technique.

\section{RELATED WORK}

Interference avoidance [6] is one of ICI mitigation strategies which deploys certain restrictions on the resources used in different cells such as restriction on frequency or power. In this section, we classify proposed avoidance algorithms into two classes: non-intercell coordination and Intercell coordination schemes and each of them can include different time scale schemes.

\subsection{Non- Intercell Coordinated Schemes}

Reuse factor one (RF1) is the simplest frequency reuse scheme in which the total available bandwidth is reused in each cell. In reuse factor three (RF3), the available bandwidth is divided into three equal orthogonal subbands and they are assigned to three neighbouring cells so that cells deploy different frequencies. In Softer Frequency Reuse (SerFR) scheme, the RF1 is used in cell edge and cell centre [7]. The main idea is to schedule RBs with more flexibility so that CEUs are allocated with a higher probability to frequency band with a higher transmit power and CCUs are assigned with a higher probability to frequency band with the lower transmit power. Some threshold values can be considered to separate CEU from CCU such as SINR, path loss, distance and so on.

In order to enhance the system performance of RF1 and RF3, the Fractional Frequency Reuse (FFR) techniques have been proposed. In FFR based schemes, the UEs who have higher signal quality use a lower reuse factor such as RF1 and UEs with the lower SINR deploy higher RF such as RF3 [8]. FFR techniques combine frequency and power allocation in different ways. Two well- 
known FFR schemes are Partial Frequency Reuse and Soft Frequency Reuse. In Partial Frequency Reuse (PFR) scheme [9], the total available bandwidth is divided into several subbands. For example, one subband is used for CCUs by using RF1 and three subbands are used for three neighbouring cells by using RF3 for CEUs. The power related to frequency of cell edge can be amplified while the total transmit power is fixed. For Soft Frequency Reuse (SFR) scheme ([1012]), the one third of available bandwidth is allocated to CEU with amplified transmit power and the rest subbands are assigned to CCU with lower transmit power. The CEUs use only the cell edge subband while CCUs have access to whole available bandwidth with lower priority than CEUs. Also different types of SFR schemes have been suggested to enhance the system performance in [13-15]. Soft Fractional Frequency Reuse (SFFR) has been introduced in [16] to enhance the total cell throughput of FFR. SFFR scheme utilizes subbands allocated to the cell edge part in the neighbouring cells with low power levels for the CCU. The difference between SFFR and SFR is that the SFFR deploys the common subband.

Although the RF1 scheme has high peak data rate, the worst ICI will be observed in this scenario, particularly at cell edge parts. RF3 leads to low ICI improvement but the large capacity is lost because only one third of the resources are used in each cell. Therefore, the worst cell throughput is obtained by RF3. In PFR, The ICI is completely eliminated for the CEUs at the cost of the spectral efficiency losses. Although in SFR, UEs have higher spectral efficiency, they suffer higher ICI because the orthogonality between cell edge and cell centre subbands of neighbouring cells is not guaranteed. There are some analyses on FFR [17-19] and but [20] has provided an analytical comparison among RF1, RF3, PFR and SFR. It has been concluded that although static schemes enhance the cell edge throughput, they might decrease the cell throughput. Because of low throughput of RF1, RF3, PFR and SFR, other algorithms have been proposed to improve the performance of LTE downlink systems. Consequently, ICI mitigation cannot effectively improve the throughput only by the power control or resource limitation especially for those UEs who are close to each other in the system [21].Therefore, some algorithms have been developed to jointly perform on both resource and power allocations to maximize the throughput.

\subsection{Intercell Coordinated Based Schemes}

In coordination based schemes, eNBs should coordinate with each other to reduce ICI. Therefore, resource allocation is performed via information exchanged on X2 interface in the eNB level without using central entity. The coordination algorithms can be divided into two subclasses: fractional frequency reuse and dynamic frequency reuse schemes.

\subsubsection{Fractional Frequency Reuse Schemes}

In these schemes the dynamic thresholds are used for CCUs' RB allocation or dynamic power allocation. In Novel Enhanced Fractional Frequency Reuse (NEFFR) [22], the total available bandwidth is split into three parts where one part is allocated to cell edge with higher power and two parts are assigned to cell centre with lower power. An interference avoidance factor (IAF) based on fairness has been introduced to balance the number of UEs and RB allocations. Dynamic Fractional Frequency Reuse (DFFR) [23] divides entire available bandwidth into three subbands. The two first subbands have maximum power while the power of third subband is determined dynamically via an interference avoidance request (IAR) message. A semi dynamic ICI avoidance scheme has been proposed in [24] which combined the advantages of static coordination and dynamic coordination. According to non-overlapping handover regions, the CEUs in each cell will be divided into several cell edge groups. A dedicated RB list is assigned to each group. For cell edge group two steps are performed to achieve the list of RBs including the static resource pre-allocation and dynamic borrowing or leasing. Reference [25] introduced a region based ICI 
International Journal of Wireless \& Mobile Networks (IJWMN) Vol. 5, No. 4, August 2013

avoidance technique in which each cell is split into some areas according to the received interferences. To split the region, some UEs thresholds and UE measurements are used. Moreover, each region has its own specific power.

\subsubsection{Dynamic Frequency Reuse Schemes}

In these schemes, the resource allocation is performed based on optimizing a function or finding the priority of UEs.

One of the powerful methods for ICI avoidance is to optimize a function. Non-cooperation game theory is a method which has been used in [26] for optimizing. The utility function selects resources which receive the least interferences from neighbouring cells. A subsequent game theory approach has been suggested in [27] for real time systems in which the virtual token approach has been added into exponential rule. In [28, 29], a utility matrix is defined to cover all three sectors of an eNB including utility matrices for each sector and utility matrices for remaining sectors which use RBs simultaneously. These utility matrices are obtained based on the possible simultaneous ICI. A Hungarian algorithm [30] is executed on utility matrix which covers all three sectors and then, a threshold based approach is applied to determine which interferers should be restricted to maximize utility matrix. A subsequent autonomic ICI algorithm has been introduced in [31]. The proposed method considered the fuzzy reinforcement learning for learning and genetic algorithm (GA) for the solution of the ICI minimization problem using Relative Narrowband TX Power (RNTP) indicator. Graph based method is another methodology to optimize the desired functions. In [32], the problem of ICI has been mapped to the graph colouring problem.

Finding the priority of UEs based on their location or traffic models is a common approach to mitigate ICI particularly for CEUs. Using gain selective way is a different approach to determine UE which has the highest chance to receive a RB. This method has been deployed in [33] and can be performed by local search or total search. In [34], a QoS approach has been suggested for multiclass services. Since different traffics have different quality of service (QoS), resources should be assigned based on different QoS rather than using the same method. For latencysensitive service, the [35] converts the QoS of a UE into the required number of RBs using size (bit) of packet, the remained scheduling time and channel capacity. Moreover, references [36-40] have reviewed some ICI mitigation algorithms proposed for LTE with more details.

Based on simulation results obtained from references, the schemes with intercell coordination have higher throughput than schemes without intercell coordination because in intercell coordinated based schemes, resource allocation is performed via information exchanged among eNBs. Moreover, among intercell coordination methods, dynamic frequency reuse schemes show better performance in terms of cell throughput and cell edge throughput because dynamic scheme can adapt instantaneously to network changes such as traffic variation or load distributions without using a priori resource partitioning. However, as a practical aspect, several factors should be considered when an algorithm would be selected as the best technique for a particular goal in real environment. There is usually a trade-off between optimal solutions on the one side and computational cost, complexity, time and overhead on the other side. When an ICI mitigation technique uses a complicated optimization method to find the best RB and power, although the system performance may increase significantly in theory, some new challenges will come up which impact on capability for real systems. For instance, high computational cost as well as high required overhead and time cause the system cannot trace the network changes and therefore cannot adapt rapidly. Consequently, the good theoretical algorithm may not be able to implement in the real world. In this paper we proposed a dynamic ICI mitigation scheme with simple fuzzy logic system to reduce the complexity. 


\section{THE PROPOSED ICIC SCHEME}

In this scheme we focus on how to assign RB to different UEs and determine the transmit power of RBs in each cell. The main objective of this scheme is to mitigate the ICI in the LTE downlink system. Note that the downlink transmission is considered because the related broadband services pose higher rate requirements than those in uplink. The proposed scheme is executed in three phases as shown in Figure. 2. In phase A, we focus on how to find priorities of UEs and then sort the UEs based on their priorities. The subband related to each priority is determined in phase $\mathrm{B}$. In phase $\mathrm{C}$, the required transmit power for each $\mathrm{RB}$ is obtained through a fuzzy logic system.

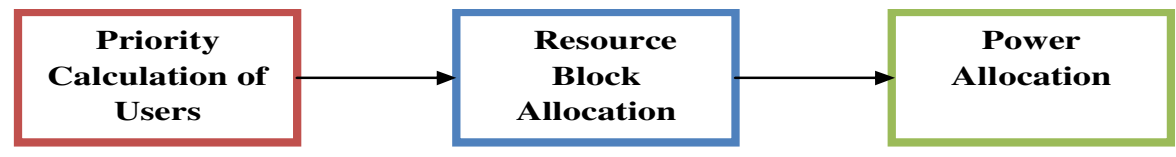

Figure 2. Overview of the proposed algorithm

\subsection{Phase A: Priority of UEs}

In phase A, we find the priority of UEs both in cell $l$ and its neighbouring cells which are going to use the same RB at the next Transmission Time Interval (TTI). When a UE comes away the serving eNB and becomes closer to a neighbouring eNB, the interference level receiving from the neighbouring eNB increases. Therefore, the UE should be scheduled on a RB which has higher transmit power in the serving cell and lower transmit power in neighbouring cells to reduce the ICI. Moreover, if different types of services are supported by the LTE system (e.g., real time and non-real time services), RBs should be allocated to different UEs based on their Quality of Service (QoS) requirements. Therefore, it is important that the QoS and Head of Line (HoL) are taken into account when the UE is selected to occupy one or more RBs. Consequently, we consider three parameters to determine the priority of UEs including interference level, QoS and HoL. Then, all parameters are normalized based on their maximum values and finally a weighting algorithm is deployed to find the final priority value.

\subsubsection{Interference Level}

In the first step, we calculate the impact of interference level, $I_{m}$, of UEm from neighbouring eNBs. Note that to calculate the interference level, an equal transmit power is considered for all eNBs on all RBs.

$$
I_{m}=\sum_{k=1}^{K} p_{m}^{n} H_{m, n}^{k}
$$

In order to calculate the maximum interference, $I_{\max }$, the following conditions is taken into account: 1) Minimum shadowing, 2) Minimum fading, 3) Maximum transmit power on the used $\mathrm{RB}$, and 4) Minimum pathloss, that is when a UE is located on the boundary of two neighbouring cells, the maximum distance from the serving eNB and minimum distance from the neighbouring cell is obtained:

$$
\text { Distance }=\frac{\left|P_{o s l}-P_{o s k}\right|}{2}
$$

where $\mathrm{Pos}_{l}$ and $\operatorname{Pos}_{k}$ are locations of serving eNB $l$ and neighbouring eNB $k$. This assumption will lead to minimum path loss received from the neighbouring eNB. By replacing these values into (2), the $I_{\max }$ will be obtained. 


\subsubsection{Head of Line (HoL)}

HoL represents the difference between the current time and the arrival time of a packet.

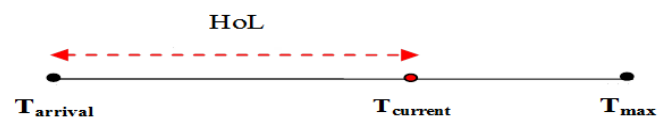

where the $T_{\text {arrival }}$ shows the time that the packet takes to arrive to the queue and $T_{\max }$ represents the maximum allowable packet delay for UEm.

\subsubsection{Quality of Service $(\mathrm{QoS})$}

QoS Class Identifier (QCI) is a scalar value considered as a reference to particular parameters that controls packet forwarding behaviours such as scheduling weights, admission thresholds, and queue management thresholds [41]. QCI is preconfigured by the operator owning the node (e.g. eNB). Note that the QCI is unique for each Service Data Flow (SDF). In addition, Guaranteed Bit Rate (GBR) is the minimum bit rate which applications request and usually deployed for applications such as Voice over Internet Protocol (VoIP). Non-GBR bearers cannot guarantee any specific bit rate, and are usually deployed for applications as web browsing.

Every QCI (GBR and Non-GBR) has a priority level in which the priority level 1 is the highest priority level. The priority levels would be deployed to differentiate between SDF aggregates of the same UE as well as SDF aggregates from different UEs [41]. We use the priority defined in Table 1 [41] as our third parameter and is represented by $Q_{m}$.

In order to combine the obtained parameters, we use a simple weighting algorithm shown in (4):

$$
\begin{gathered}
W=w_{1} \times I_{m}^{\prime}+w_{2} \times \mathrm{Hol}_{m}^{\prime}+w_{3} \times Q_{m}^{\prime} \\
\sum_{i=1}^{3} w_{i}=1
\end{gathered}
$$

Where $w_{i}$ is a coefficient corresponding to each parameter. Parameters, , and represent the normalized values of the interference level, HoL and QCI priority of UEm, respectively. These normalized values are calculated as follows:

$$
\begin{aligned}
& I_{m}^{\prime}=\frac{I_{m}}{I_{\max }} \\
& H o L_{m}^{\prime}=\frac{H o L_{m}}{T_{\max }} \\
& Q_{m}^{\prime}=\frac{1}{Q_{m}}
\end{aligned}
$$

\subsection{Phase B: Resource Block Allocation}

The bandwidth division is based on UEs priority. The total available bandwidth $B W$ is divided into three non-overlapping subbands shown in Figure 3 (a): 1) High Subband (HS): the subband which is allocated to UEs with higher priority; 2) low Subband $(L S)$ : the subband assigned to

Table 1. Standardized QCI characteristics [41] 
International Journal of Wireless \& Mobile Networks (IJWMN) Vol. 5, No. 4, August 2013

\begin{tabular}{|c|c|c|c|c|c|}
\hline QCI & $\begin{array}{l}\text { Resource } \\
\text { Type }\end{array}$ & Priority & $\begin{array}{l}\text { Packet Delay } \\
\text { Budget }\end{array}$ & $\begin{array}{l}\text { Packet Error } \\
\text { Loss Rate }\end{array}$ & Example Services \\
\hline 1 & \multirow{4}{*}{ GBR } & 2 & $100 \mathrm{~ms}$ & $10^{-2}$ & Conversational Voice \\
\hline 2 & & 4 & $150 \mathrm{~ms}$ & $10^{-3}$ & $\begin{array}{l}\text { Conversational Video } \\
\text { (Live Streaming) }\end{array}$ \\
\hline 3 & & 3 & $50 \mathrm{~ms}$ & $10^{-3}$ & Real Time Gaming \\
\hline 4 & & 5 & $300 \mathrm{~ms}$ & $10^{-6}$ & $\begin{array}{l}\text { Non-Conversational } \\
\text { Video (Buffered } \\
\text { Streaming) }\end{array}$ \\
\hline 5 & \multirow{5}{*}{ Non-GBR } & 1 & $100 \mathrm{~ms}$ & $10^{-6}$ & IMS Signalling \\
\hline 6 & & 6 & $300 \mathrm{~ms}$ & $10^{-6}$ & $\begin{array}{l}\text { Video (Buffered } \\
\text { Streaming) } \\
\text { TCP-based }\end{array}$ \\
\hline 7 & & 7 & $100 \mathrm{~ms}$ & $10^{-3}$ & $\begin{array}{l}\text { Voice, Video (Live } \\
\text { Streaming), Interactive } \\
\text { Gaming }\end{array}$ \\
\hline 8 & & 8 & \multirow{2}{*}{$300 \mathrm{~ms}$} & \multirow{2}{*}{$10^{-6}$} & Video (Buffered \\
\hline 9 & & 9 & & & $\begin{array}{l}\text { Streaming) } \\
\text { TCP-based }\end{array}$ \\
\hline
\end{tabular}

UEs with lower priority, and 3) Common Subband (CS): the subband which can be allocated to all UEs with different types of priority. Therefore, RB allocation for UE with lower priority in $L S$ does not affect the number of RBs that can be allocated to UEs with highest priority in $H S$.

After priority calculation, UEs in each cell as well as UEs in neighbouring cells which use the same RB should be sorted based on their priority. For this purpose, we first sort the UEs which are located in one cell. The UE with the highest priority is put in the first location of the queue. Then, the suitable RB from the relevant subband is allocated to each UE. In $H S$, the RB allocation is started by the UE with the highest priority while in $L S$ the UE with the lowest priority is selected at first. Since the $L S$ of one cell is selected as $H S$ for another cell, it is possible that the $\mathrm{UE}$ in $H S$ request the neighbouring eNB to reduce the transmit power on that $\mathrm{RB}$ in order to reduce the impacted interference. In addition, this approach allows us to use the remaining transmit power from $L S$ for $H S$ under the specified conditions to improve the throughput while the total transmit power is still smaller than or equal to the maximum transmit power of eNB. More details about transmit power will be described in power allocation.

\subsection{Phase C: Power Allocation}

Using a higher/lower transmit power by neighbouring eNBs on a same RB can directly lead to increase or decrease of UE's received signal levels. It will change the interference level impacted on UE by neighbouring eNB. The key idea of the proposed power allocation algorithm is that eNBs dynamically control the transmit power using fuzzy logic system (FLS) [42] and exchanging messages with each other. This algorithm can be executed for several TTIs until the power changes become small and after that it will be executed periodically or by a trigger.

After calculating the priority of UEs who are selected as a candidate to use a same RB (Figure. 3 (b)), the transmit power of each eNB on that RB would be computed. For example, as we can see in Table 2, UE44 is located in $L S$ of cell 1 while UE1 is in $H S$ of cell 3. If the serving eNB of UE44 (eNB1) results highest interference on UE1 so that the SINR of UE1 becomes very low, 

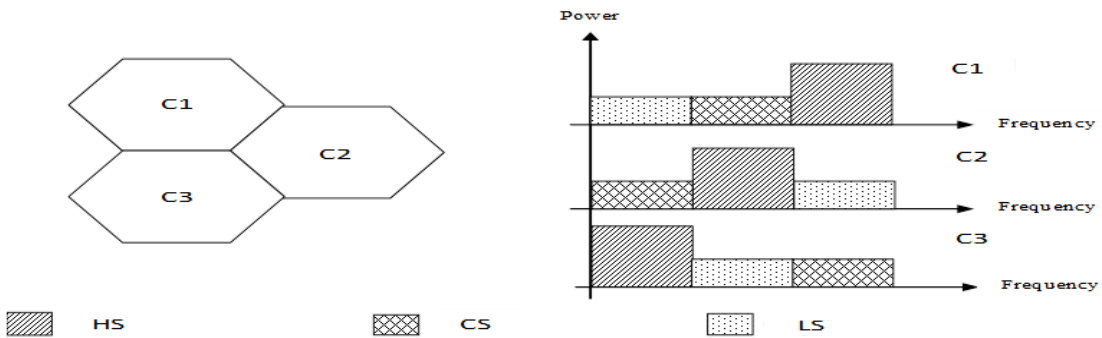

(a)

\begin{tabular}{|c|c|c|c|c|c|c|c|}
\hline & \multicolumn{6}{|c|}{ Priority } \\
\hline & & \multicolumn{2}{|c|}{ Highest } & \multicolumn{4}{|c|}{ 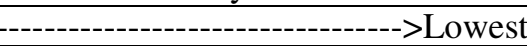 } \\
\hline \multirow{3}{*}{ Queue } & Cell 3 & UE1 & UE2 & UE3 & $\ldots$ & UE14 & UE15 \\
\hline & Cell 2 & UE16 & UE17 & UE18 & $\ldots$ & UE29 & UE30 \\
\hline & Cell1 & UE31 & UE32 & UE33 & & UE43 & UE44 \\
\hline
\end{tabular}

(b)

Figure 3. (a) Bandwidth division, (b) locations of UEs in queues of different cells based on UE's priority

serving eNB of UE1 (eNB3) requests eNB1 to decrease its transmit power. In the other word, the strongest interferes have to decrease their transmit powers on a given RB if their UEs have lower priority on that $\mathrm{RB}$ while the minimum achievable data rate is satisfied. It is possible that one eNB has to decrease the transmit power of one RB several times, because its UEs has lower priority than UEs of neighbouring cells and it is detected as a strongest interferer for some neighbouring cells. In this case, the minimum obtained transmit power is selected as a transmit power for that RB. Moreover, if the total transmit power of eNB3 is smaller than the maximum transmit power, eNB3 can increase the transmit power on RB1. The amount of power decreasing or increasing could be obtained through FLS.

Table2. Allocating UEs to subbands for different cells based on UE's priority

\begin{tabular}{|c|c|c|c|c|c|c|c|c|c|c|c|c|c|}
\hline Cell & $\mathrm{RB1}$ & $\mathrm{RB} 2$ & $\ldots$ & $\mathrm{RB}_{\mathrm{n} / 3}$ & $\mathrm{RB}_{(\mathrm{n} / 3)+1}$ & $\mathrm{RB}_{(\mathrm{n} 3)+2}$ & $\ldots$ & $\mathrm{RB}_{(2 \mathrm{n} 3)}$ & $\mathrm{RB}_{(2 \mathrm{n} 3)+1}$ & $\mathrm{RB}_{(2 \mathrm{n} 3)+2}$ & $\ldots$ & $\begin{array}{c}\mathrm{RB}_{\mathrm{Il}} \\
1\end{array}$ & $\mathrm{RB}_{\mathrm{n}}$ \\
\hline 3 & $\mathrm{UE} 1$ & $\mathrm{UE} 2$ & $\ldots$ & $\ldots$ & $\mathrm{U} 15$ & $\mathrm{U} 14$ & $\ldots$ & $\ldots$ & $\ldots$ & $\ldots$ & $\ldots$ & $\ldots$ & $\ldots$ \\
\hline 2 & $\ldots$ & $\ldots$ & $\ldots$ & $\ldots$ & $\mathrm{UE} 16$ & $\mathrm{UE} 17$ & $\ldots$ & $\ldots$ & $\mathrm{UE} 30$ & $\mathrm{UE} 29$ & $\ldots$ & $\ldots$ & $\ldots$ \\
\hline 1 & $\mathrm{UE} 44$ & $\mathrm{UE} 43$ & $\ldots$ & $\ldots$ & $\ldots$ & $\ldots$ & $\ldots$ & $\ldots$ & $\mathrm{UE} 31$ & $\mathrm{UE} 32$ & $\ldots$ & $\ldots$ & $\ldots$ \\
\hline$\ldots$ & $\ldots$ & $\ldots$ & $\ldots$ & $\ldots$ & $\ldots$ & $\ldots$ & $\ldots$ & $\ldots$ & $\ldots$ & $\ldots$ & $\ldots$ & $\ldots$ & $\ldots$ \\
\hline
\end{tabular}

In order to determine the transmit power of each eNB on a particular RB, we use the FLS [42] because its design is simple and can work on time [43]. A FLS is an expert system using "IF... THEN" rules and includes very simple concept. It could simultaneously work with numerical data and linguistic information using a nonlinear mapping between input data and scalar output data. Since FLS deploys linguistic terms, the previous information can be gathered easier using the experience of an operator. The main difference between FLS and conventional rule base controller is that FLS can simultaneously trigger several rules which lead to smoother control. In general, FLS includes three steps shown in Figure 4:

1. Input step: it maps inputs to the appropriate membership functions and truth values.

2. Processing step: this step selects appropriate rules and finds their results. Then, it combines the results of the chosen rules. 
3. Output step: in this step the obtained results are converted to a specific output value.

Through FLS, several states and actions could be defined. The SINR, historical throughput and achievable data rate are selected as the inputs of fuzzy interface because they are important metrics in LTE downlink system which can monitor the UE as well as system performances:

1) SINR of UE with higher priority.

2) Historical throughput of UE with lower priority.

3) The current achievable data rate that UE with lower priority could be achieved if it uses that RB. Each cell checks the obtained achievable data rate from its neighbouring cells through a data rate indicator. It is 2 if the achieved data rate is more than minimum achievable data rate, value 1 represents only the minimum achievable data rate is satisfied otherwise it is 0 .

The crisp output of FLS is the transmit power of UEs which can change as follows:

1) Transmit power of UE with lower priority (i.e., $L S$ or $C S$ ) could be fixed, avoided or decreased.

2) Transmit power of UE with higher priority (i.e., $H S$ ) might increase or be fixed.

In the inference stage, mapping of inputs to outputs are defined by a set of "IF-Then" rules. In the defuzzification stage, the output value is achieved using the aggregation of all rules and the centre

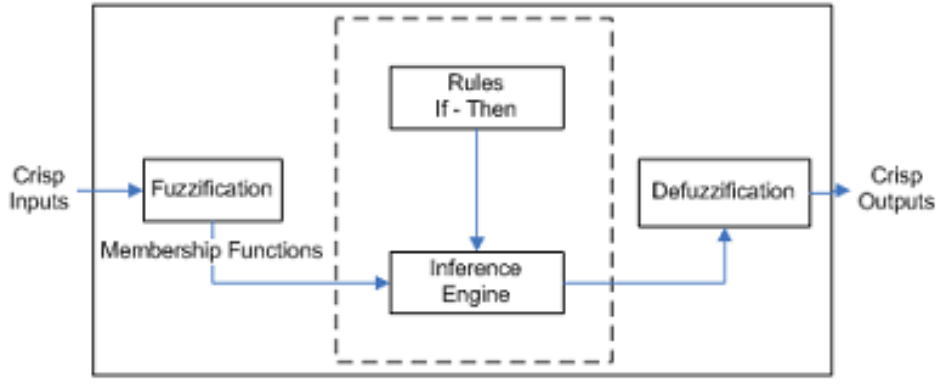

Figure 4. Overview of fuzzy logic system

of gravity approach. By implementation of FLS, the final transmit power for each RB in each cell would be obtained.

\section{SIMULATION RESULTS}

In order to evaluate the performance of the proposed priority scheme and compare with the wellknown schemes, RF1 and SFR schemes, we describe the simulation results in this section.

\subsection{Simulation Setup}

Assessment is based on the system level simulation in a LTE downlink network with 19 cells. The network topology is composed of a set of cells and network nodes including macro eNBs and UEs which are distributed within cells. A certain area is defined for simulation where the eNBs and UEs are located and only in this region, UE movement and transmission are simulated. Each network node is identified by a unique ID and its position is defined using Cartesian system. A wraparound function should be used to make sure that the users do not exit from the simulation 
area. The simulation length is measured by TTIs which equals $1 \mathrm{~ms}$. The simulation assumptions are outlined in Table 3. In addition, a streaming video is considered as the traffic model.

In order to consider power spectral density mask limitations, the power allocation is started based on SFR schemes. The initial transmit power of $H S$ is presented by $P_{H}$ and set to $\alpha \frac{P_{T}}{N}$ while the initial transmit power for both $L S, P_{L}$, and for $C S, P_{C}$, equal $\frac{(3-\alpha)}{2} \frac{P_{T}}{N} \quad(\alpha>1)$. Note that $P_{T}$ is total transmit power of each cell and $N$ is number of RBs. Moreover, a FLS has been implemented and a set of "IF-Then" rules defines the mapping of inputs to output including 27 rules for each output. For example "If SINR is low and the historical throughput is high and the achievable data rate is high Then the transmit power of $L S$ reduces" or "If SINR is low and historical throughput is high and achievable data rate is high Then the transmit power of $H S$ increases". The membership functions for inputs and outputs are specified as follows:

Table 3. Simulation parameters

\begin{tabular}{|l|l|}
\hline Parameter & Assumption \\
\hline Cellular layout & Hexagonal grid, 19 cells, wrap around \\
\hline Inter-site distance & $500 \mathrm{~m}$ \\
\hline Carrier frequency & $2 \mathrm{GHz}$ \\
\hline Carrier bandwidth & $5 \mathrm{MHz}$ \\
\hline Channel model & Rayleigh \\
\hline Shadow Fading & Log-normal \\
\hline Path loss model & $128.1+37.6 \log (\mathrm{R}), \mathrm{R}$ in $\mathrm{km}$ \\
\hline Total macro Tx power & $43 \mathrm{dBm}$ \\
\hline UE speed & $3 \mathrm{~km} / \mathrm{h}$ \\
\hline Scheduler & Round Robin \\
\hline$\alpha$ & 2 \\
\hline
\end{tabular}

1) Each input is fuzzified using three membership functions named "Low", "Medium" and "High" which are shown by "Z-shaped", "Triangular-shaped" and "S-shaped" respectively.

- The range of membership functions is fixed for all SINR inputs according to SINR range obtained from SINR-Block Error Rate (BLER) curves [44].

- The range of membership functions is fixed for all achievable data rate inputs according to Channel Quality Indicator (CQI) efficiency [44].

- For throughput, the range of membership function could be changed based on the average number of RBs allocated to each UE in each cell.

2) To fuzzify the outputs, two different scenarios are assumed:

- For transmit power of UE which belongs to $L S$ and $C S$, the membership function of output is change from zero to maximum transmit power per RB. The membership functions are tagged with "Avoid", "Fix" and "Decrease".

$>$ For transmit power of UE in $H S$, membership functions are named by "Fix" and "Increase". The range of membership functions are changed from initial power to maximum transmit power plus to $\Delta P$. Note that $\Delta P$ represents the additional transmit power which is obtained from reducing transmit power on other RBs for each cell.

Note that, in this paper the average cell throughput is defined as the amount of data sent successfully in a cell over a period of time (i.e., one TTI). Moreover, 5\% edge throughput is considered as the $5^{\text {th }}$ percentile point of the cumulative distribution function (CDF) of the user throughput which indicates the minimum throughput achieved by $95 \%$ of UEs [45].Cell edge user throughput means the average throughput of UEs located in cell edge regions. The results of the proposed priority scheme will be compared with RF1 and SFR schemes. 


\subsection{Performance Evaluation}

As mentioned in Section 4, the RB allocation is based on a weighing algorithm with three coefficients including $w_{1}, w_{2}$ and $w_{3}$. Therefore, the difference in coefficient values can lead to different results as shown in Table 4. For example, when the coefficient value of interference level, $w_{1}$, is higher than $w_{2}$ and $w_{3}$, the interference level of UEs decreases which leads to improve the throughput. However, the delay increases because the UEs who have the higher interference level (i.e., CEUs) are selected as highest priority instead of UEs with larger delay. In this case, the interference level has higher priority than delay and type of service. On the other hands, when the highest coefficient value is allocated to delay, although the delay of system decreases, the throughput is reduced. Consequently, the coefficient values affect the system performance and they should precisely be specified.

In order to show the performance of system clearly in terms of delay and interference level, the simulation results obtained by the proposed priority scheme are compared with RF1 and SFR. It is obvious that the proposed priority scheme can reduce the delay because the delay is one factor of

Table 4. System performance comparison for different coefficient values

\begin{tabular}{|c|c|c|c|c|c|c|}
\hline $\mathbf{W}_{\mathbf{1}}$ & $\mathbf{W}_{\mathbf{2}}$ & $\mathbf{W}_{\mathbf{3}}$ & $\begin{array}{c}\text { Cell Edge } \\
\text { Throughput } \\
{[\mathbf{K b p s}]}\end{array}$ & $\begin{array}{c}\text { Mean Cell } \\
\text { Throughput } \\
{[\mathbf{M b p s}]}\end{array}$ & $\begin{array}{c}\text { Delay } \\
{[\mathbf{m s}]}\end{array}$ & $\begin{array}{c}\text { Interference } \\
{[\mathbf{d B}]}\end{array}$ \\
\hline 0.33 & 0.33 & 0.33 & 233.92 & 8.64 & 10.13 & -89.06 \\
\hline 0.2 & 0.6 & 0.2 & 230.84 & 8.48 & 11.35 & -88.94 \\
\hline 0.2 & 0.2 & 0.6 & 209.07 & 8.40 & 9.88 & -89.08 \\
\hline 0.6 & 0.2 & 0.2 & 274.55 & 9.14 & 11.24 & -87.83 \\
\hline
\end{tabular}

weighting algorithm (see Figure 5). When the HoL of a UE increases, its priority increases and then it will be scheduled to transmit data with higher Modulation and Coding Scheme. Moreover, Figure 6 demonstrates that the interference level of the proposed priority scheme is lower than RF1 and SFR because the transmit power of each RB is calculated dynamically by exchanging messages among neighbouring eNBs. As a result, the interference level on each RB in each cell decreases.

The Figure 7 depicted that the proposed priority scheme can significantly improve the $5 \%$ of UE's throughput than RF1 and SFR due to considering interference level. In addition, Figure 8 demonstrates that the proposed priority scheme has better cell edge user throughput than RF1 and SFR schemes. Simulation result shows that the cell edge throughout of the proposed priority scheme is $68 \%$ higher than SFR and $96 \%$ higher than RF1.

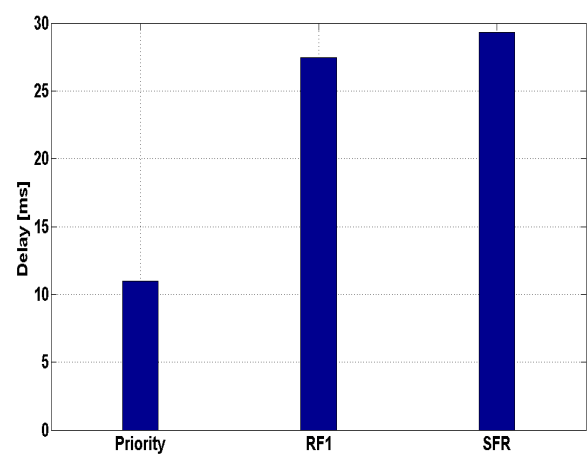

Figure 5. Delay comparison among priority, RF1 and SFR schemes

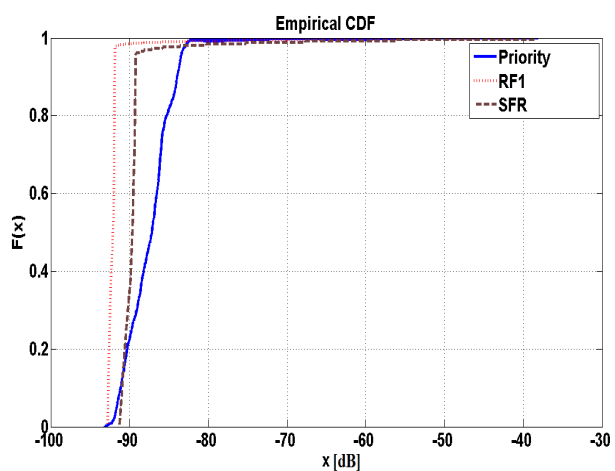

Figure 6. Interference level comparison among priority, RF1 and SFR schemes 
In the proposed priority scheme, real time coordination is performed among all involved cells to avoid the use of same RB in two CEU in neighbouring cells. This is because the interference level of UEs has been considered as a parameter to find the priority. Moreover, unlike the RF1 and SFR schemes, the transmit power allocated to each UE is dynamically calculated through a FLS. The dynamic power allocation can lead to a reduction of interference for UEs particularly for CEU. Therefore, SINR increases and then a higher CQI value is selected. On the other side, CEUs and CCUs can be scheduled within the available bandwidth not only in a specified subband such as SFR. It leads to increase in the number of RBs which can be allocated to each UE. In RF1 and SFR the RB and power allocation in each cell is pre-defined and not adapted to the changes in network traffic.

Figure 9 compares the average cell throughput of the proposed priority scheme with RF1 and SFR. It shows that the proposed priority scheme has higher average system performance than SFR and RF1 around $12 \%$ and $28.7 \%$ respectively. This is because it dynamically adjusts the transmit power of each RB based on SINR, throughput and achievable data rate through the FLS. Moreover, it takes into account the system traffic loads as well as system changes when the RBs are allocated to different UEs.

\section{CONCLUSION}

A priority based ICIC scheme has been proposed in this paper to mitigate the ICI problem in LTE downlink system. The proposed priority scheme determines the priority of each UE using interference level, Quality of Service and Head of Line. UEs could be scheduled based on their priority on the specified subbands. Therefore, the network changes and traffic model can be considered in this method. The adaptive transmit power is determined through a fuzzy logic system. Simulation results showed that the proposed priority scheme outperforms the RF1 and SFR schemes in terms of cell edge user throughput and system throughput. As the future work, we will propose an enhanced intercell interference coordination algorithm to mitigate interference in LTE-Advanced heterogeneous networks.

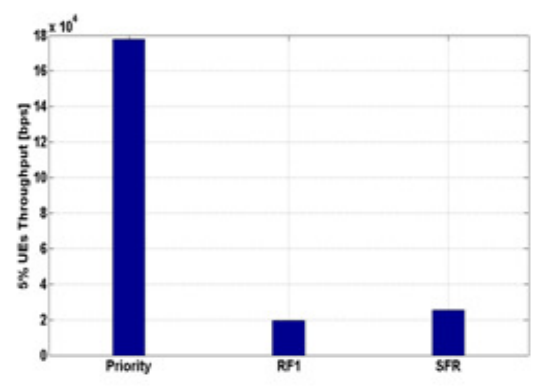

Figure $7.5 \%$ UE throughput comparison

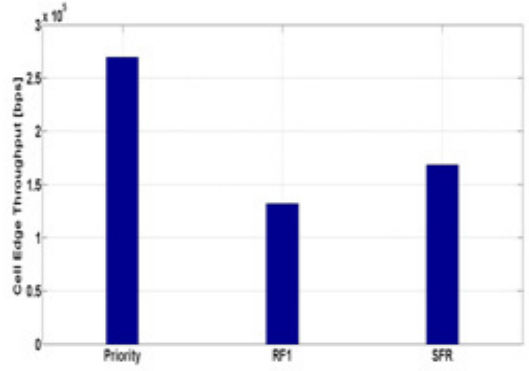

Figure 8. Average cell edge user throughput comparison

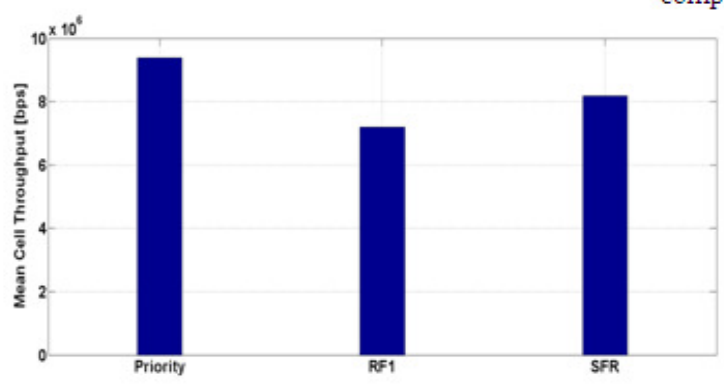

Figure 9. Average cell throughput comparison 
International Journal of Wireless \& Mobile Networks (IJWMN) Vol. 5, No. 4, August 2013

\section{REFERENCES}

[1] I.F. Akyildiz, D. M. G. Estevez, E.Ch.Reyes, (2010) "The evolution to 4G cellular systems: LTEAdvanced", Physical Communication, Vol.3, No.4, pp. 217-244.

[2] F. Khan, (2009) . LTE for 4G Mobile Broadband - overview of Inter-Cell Interference Coordination in LTE, United States of America by Cambridge University Press.

[3] C. Gessner, (2008).UMTS Long Term Evolution (LTE) Technology Introduction,Rohde \& Schwarz Products.

[4] D. Astely, E. , Dahlman, A. Furuskar, Y. Jading, M. Lindstrom, and S. Parkvall, (2009) "LTE: the evolution of mobile broadband", IEEE Communications Magazine, Vol.47, No.4, pp.44-51.

[5] E. Lawrey, (1999) "multiuser OFDM", IEEE International Symposium on signals Processing and its Applications, Vol.2, pp. 761-764.

[6] F.Khan, (2009).LTE for 4G Mobile Broadband: Air Interace Technologies and performance, Cambridge University Press.

[7] X. Zhang, Ch.He, L.Jiang, and J.Xu , (2008) "Inter-cell interference coordination based on softer frequency reuse in OFDMA cellular systems", International Conference on Neural Networks and Signal Processing, pp.270 - 275.

[8] Siemens, R1-060135, (2006) "Interference Mitigation by Partial Frequency Reuse", 3GPP RAN WG1\#42, London, UK.

[9] Huawei, R1-050507, (2005) "Soft Frequency Reuse Scheme for UTRANLTE”, 3GPP RAN WG1\#41, Athens, Greece.

[10] Huawei, (2005) "Further Analysis of Soft Frequency Reuse Scheme".

[11] Huawei, (2005) "Soft Frequency Reuse Scheme for UTRAN LTE".

[12] Ericsson, R1-061374, (2006)“Downlink intercell interference coordination/avoidance evaluation of frequency reuse”, 3GPP Project Document.

[13] R. Ghaffar and R. Knopp, (2010) "Fractional frequency reuse and interference suppression for OFDMA networks", 8th International Modelling and Optimization in Mobile, Ad Hoc and Wireless, pp. $273-277$.

[14] F.B. Mugdim, (2007)"Interference Avoidance Concepts," WINNER II project.

[15] E. Haro, S. Ruiz, D. Gonzalez, M.G-Lozano, and J. Olmos, (2009).Comparison of Different Distributed Scheduling Strategies for Static/Dynamic LTE Scenarios, Technical University of Wien.

[16] IEEE 802.16 Broadband, (2008) "Fractional Frequency Reuse in Uplink", LG Electronics.

[17] M. Porjazoski and B. Popovski, (2010) "Analysis of Intercell interference coordination by Fractional frequency reuse in LTE", in Proc. Int Software, Telecommunications and Computer Networks (SoftCOM) Conference, pp. 160-164.

[18] M. Porjazoski and B. Popovski, (2010) "Contribution to analysis of Intercell interference coordination in LTE: A fractional frequency reuse case", Global Mobile Congress (GMC), pp.1-4.

[19] A. Mills, D. Lister and M. DeVos, (2011) "Understanding Static Inter-cell Interference Coordination Mechanisms in LTE”, Journal of Communications, Vol.6, No.4, pp. 312-318.

[20] S-E. Elayoubi, O. BenHaddada, and B. Fouresti'e, (2008) "Performance Evaluation of Frequency Planning Schemes in OFDMA based Networks", IEEE Transaction on Wireless Communications, Vol.7, No.5, pp.1623-1633.

[21] Y.Yu, E. Dutkiewicz, X. Huang, and M. Mueck, (2012) “ A Resource Allocation Scheme for Balanced Performance Improvement in LTE Networks with Intercell Interference” IEEE Wireless Communication and Networking conference: MAC and Cross-layer Design, pp. 1630-1635.

[22] L. Liu, G. Zhu, D. Wu, (2011) “A Novel Fractional Frequency Reuse Structure Based on Interference Avoidance Scheme in Multi-cell LTE Networks", 6th International ICST Conference on Communications and Networking in China (CHINACOM), pp.551 - 555.

[23] L. Liu, D. Qu, and T. Jiang, (2010) "Dynamic Fractional Frequency Reuse based on Interference Avoidance Request for Downlink OFDMA Cellular Networks", IWCMC, Caen, France, pp.381-386.

[24] L. Dong, and W. Wenbo, (2009) "A Novel Semi-Dynamic Inter-Cell Interference Coordination Scheme Based on UE Grouping”, IEEE 70th Vehicular Technology Conference, pp.1-5.

[25] Ch.You, Ch.Seo, Sh. Portugal, G. Park, T. Jung, H. Liu and I. Hwang, (2011) "Intercell Interference Coordination Using Threshold-Based Region Decisions," Wireless Personal Communications, Vol. 59, No.4, pp.789-806. 
International Journal of Wireless \& Mobile Networks (IJWMN) Vol. 5, No. 4, August 2013

[26] L. Shuhui, C. Yongyu, Y. Ruiming, and Y. Dacheng, (2011) "Efficient Distributed Dynamic Resource Allocation for LTE Systems", Vehicular Tecnology conference (VTC), pp.1-5.

[27] M. Iturralde, A. Wei, T-Ali. Yahiya, and A-L. Beylot, (2012) "Resource allocation for real time services using cooperative game theory and a virtual token mechanism in LTE networks", IEEE Conference on Consumer Communications and networking (CCNC), pp.879-883.

[28] M. Rahman, and, H. Yanikomeroglu, (2010) "Enhancing cell-edge performance: a downlink dynamic interference avoidance scheme with intercell coordination", IEEE Transaction on Wireless Communications, Vol.9, No.4, pp.1414-1425.

[29] M. Rahman, H. Yanikomeroglu, and W. Wong, (2009) "Interference Avoidance with Dynamic InterCell Coordination for Downlink LTE System”, IEEE Wireless Communications and Networking Conference (WCNC), pp.1-6.

[30] H.W. Khun, (1995) "The Hungarian method for the assignment problem," Naval Research Logistic Quarterly, Vol. 2, pp. 83-97,

[31] P. Vlacheas, E. Thomatos, K. Tsagkaris, and P. Demestichas, (2011)“Autonomic downlink inter-cell interference coordination in LTE Self-Organizing Networks", 7th International Conference on Network and Service Management (CNSM), pp.1-5.

[32] M.C. Necker, (2009) "Scheduling Constraints and Interference Graph Properties for Graph-based Interference Coordination in Cellular OFDMA Networks", Journal of Mobile Networks and Applications, Vol.14, No.4, pp.539-550.

[33] G.I.Fraimis , V. D.Papoutsis, and S.A. Kotsopoulos, (2010) “A distributed radio resource allocation algorithm with interference coordination for multi-cell OFDMA systems", IEEE 21st International Symposium on Personal Indoor and Mobile Radio Communications (PIMRC), pp. 1354 - 1359.

[34] Q. Ai, P. Wang, F. Liu, Y. Wang, F. Yang and J. Xu, (2010) "QoS-guaranteed cross-layer resource allocation algorithm for multiclass services in downlink LTE system”, International Conference on Wireless Communications and Signal Processing (WCSP), pp.1-4.

[35] Z. Bingbing, C. Liquan, Y. Xiaohui, and W. Lingling, (2010) "A Modified Inter-Cell Interference Coordination Algorithm in Downlink of TD-LTE", 6th International Conference on Wireless Communications Networking and Mobile Computing (WiCOM), pp.1-4.

[36] Kh. Elsayed, (2011) "4G++: Advanced Performance Boosting Techniques in 4th Generation Wireless Systems and Beyond", The First NTRA Knowledge Dissemination and Networking Conference, Cairo, Egypt.

[37] G. Boudreau, J. Panicker, N. Guo, R. Chang, N. Wang, and S. Vrzic, Nortel, (2009) "Interference Coordination and Cancellation for 4G Networks", IEEE Communications Magazine, Vol. 47, No. 4, pp. $74-81$.

[38] R. Kwan and C. Leung, (2010) “A Survey of Scheduling and Interference Mitigation in LTE,” Journal of Electrical and Computer Engineering, Vol.2010, pp.1-10.

[39] N. Himayat, Sh. Talwar, A. Rao and R. Soni, (2010) "Interference management for 4G cellular standards", IEEE Communications Magazine,Vol.48, No.8, pp.86-92.

[40] E. Pateromichelakis, M. Shariat, A. Quddus, and R.Tafazolli, (2012) “ On the Evalution of multi-cell Scheduling in 3GPP LTE/LTE-A”, IEEE Communication Surveys and Tutorials, issue.99, pp.1-17.

[41] 3GPP, TS 23.203, (2012) "Digital cellular telecommunications system (Phase 2+); Universal Mobile Telecommunications System (UMTS); LTE; Policy and charging control architecture", V10.6.0.

[42] L. Zadeh, (1965)“Fuzzy sets”, Information Control, Vol. 8,No.3, pp.338-353.

[43] T. Ross, (1995). Fuzzy Logic with engineering application, McGraw-Hill.

[44] S. Sesia, I. Toufik, M. Baker, (2011). Lte - The Umts Long Term Evolution: From Theory to Practice, Wiley, pp. 1-648, ISBN: 0470697164, 9780470697160.

[45] G. Roche, A. Al-Glazunov, and B. Allen, (2012). LTE-Advanced and Next Generation Wireless Networks: Channel Modelling and Propagation, Willey, pp.1-566, ISBN: 978-1-1199-7670-7.

\section{Authors}

Ameneh Daeinabi is a $\mathrm{PhD}$ student in Telecommunications Engineering at University of Technology Sydney, Australia and her current research area is 4G mobile networks. She received her M.Sc. degree in Telecommunications Engineering from Sahand University of Technology in 2009 and her B.Sc. in Electrical \&Electronic Engineering from Azad University, in 2005. Her main research interests include 4G mobile networks and Vehicular Ad hoc Networks. 
International Journal of Wireless \& Mobile Networks (IJWMN) Vol. 5, No. 4, August 2013

Kumbesan Sandrasegaran is an Associate Professor at UTS and Director of the Centre for Real-Time Information Networks (CRIN). He holds a $\mathrm{PhD}$ in Electrical Engineering from McGill University (Canada)(1994), a Master of Science Degree in Telecommunication Engineering from Essex University (1988) and a Bachelor of Science (Honours) Degree in Electrical Engineering (First Class) (1985). He was a recipient of the Canadian Commonwealth Fellowship (1990-1994) and British Council Scholarship (1987-1988). His current research work focuses on two main areas (a) radio resource management in mobile networks, (b) engineering of remote monitoring systems for novel applications with industry through the use of embedded systems, sensors and communications systems. He has published over 100 refereed publications and 20 consultancy reports spanning telecommunication and computing systems.

Xinning Zhu is an associate professor at School of Information and Communication Engineering, BUPT, China. She received her PhD, MS and BS degree in Communication and Information system from Beijing University of Posts and Telecommunications (BUPT) in 2010, 1995 and 1992. Her current research interests focus on interference management and mobility management in radio resource management for heterogeneous networks. 